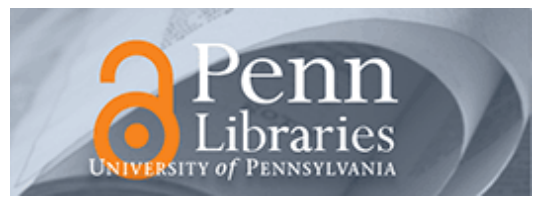

University of Pennsylvania

ScholarlyCommons

Statistics Papers

Wharton Faculty Research

1980

\title{
The FKG Inequality and Some Monotonicity Properties of Partial Orders
}

Larry A. Shepp

University of Pennsylvania

Follow this and additional works at: https://repository.upenn.edu/statistics_papers

Part of the Applied Mathematics Commons, and the Statistics and Probability Commons

Recommended Citation

Shepp, L. A. (1980). The FKG Inequality and Some Monotonicity Properties of Partial Orders. SIAM Journal on Algebraic Discrete Methods, 1 (3), 295-299. http://dx.doi.org/10.1137/0601034

This paper is posted at ScholarlyCommons. https://repository.upenn.edu/statistics_papers/418

For more information, please contact repository@pobox.upenn.edu. 


\title{
The FKG Inequality and Some Monotonicity Properties of Partial Orders
}

\author{
Abstract \\ Let $\left(a_{1}, \ldots, a_{m}, b_{1}, \ldots, b_{n}\right)$ be a random permutation of $1,2, \ldots, m+n$. Let $P$ be a partial order on the a's \\ and $b^{\prime}$ ' involving only inequalities of the form $a_{i}<a_{j}$ or $b_{i}<b_{j}$, and let $P^{\prime}$ be an extension of $P$ to include \\ inequalities of the form $a_{i}<b_{j}$; i.e, $P^{\prime}=P \cup P^{\prime \prime}$, where $P^{\prime \prime}$ involves only inequalities of the form $a_{j}<b_{j}$. We \\ prove the natural conjecture of R. L. Graham, A. C. Yao, and F. F. Yao [SIAM J. Alg. Discr. Meth. 1 (1980), \\ pp. 251-258] that in particular $\left(^{*}\right) \operatorname{Pr}\left(a_{1}<b_{1} \mid P^{\prime}\right) \geq \operatorname{Pr}\left(a_{1}<b_{1} \mid P\right)$. We give a simple example to show that \\ the more general inequality $\left(^{*}\right)$ where $P$ is allowed to contain inequalities of the form $a_{i}<b_{j}$ is false. This is \\ surprising because as Graham, Yao, and Yao proved, the general inequality $\left(^{*}\right)$ does hold if $P$ totally orders \\ both the a's and the $b$ 's separately. We give a new proof of the latter result. Our proofs are based on the \\ FKG inequality. \\ Disciplines \\ Applied Mathematics | Statistics and Probability
}




\title{
THE FKG INEQUALITY AND SOME MONOTONICITY PROPERTIES OF PARTIAL ORDERS*
}

\author{
L. A. SHEPP $\dagger$
}

\begin{abstract}
Let $\left(a_{1}, \cdots, a_{m}, b_{1}, \cdots, b_{n}\right)$ be a random permutation of $1,2, \cdots, m+n$. Let $P$ be a partial order on the $a$ 's and $b$ 's involving only inequalities of the form $a_{i}<a_{j}$ or $b_{i}<b_{j}$, and let $P^{\prime}$ be an extension of $P$ to include inequalities of the form $a_{i}<b_{j}$; i.e., $P^{\prime}=P \cup P^{\prime \prime}$, where $P^{\prime \prime}$ involves only inequalities of the form $a_{i}<b_{j}$. We prove the natural conjecture of R. L. Graham, A. C. Yao, and F. F. Yao [SIAM J. Alg. Discr. Meth. 1(1980), pp. 251-258] that in particular $\left(^{*}\right) \operatorname{Pr}\left(a_{1}<b_{1} \mid P^{\prime}\right) \geqslant \operatorname{Pr}\left(a_{1}<\right.$ $\left.b_{1} \mid P\right)$. We give a simple example to show that the more general inequality $(*)$ where $P$ is allowed to contain inequalities of the form $a_{i}<b_{j}$ is false. This is surprising because as Graham, Yao, and Yao proved, the general inequality $\left({ }^{*}\right)$ does hold if $P$ totally orders both the $a$ 's and the $b$ 's separately. We give a new proof of the latter result. Our proofs are based on the FKG inequality.
\end{abstract}

1. Introduction. Suppose $\left(a_{1}, a_{2}, \cdots, a_{m}, b_{1}, \cdots, b_{n}\right)$ is a random (uniformly distributed) permutation of $1,2, \cdots, m+n$. Following [GYY], we might think of the permutation as the actual ranking of the tennis skill of players $a_{1}, \cdots, a_{m}, b_{1}$, $\cdots, b_{n}$. Here player $x$ always loses to player $y$ in a match if $x<y$. In a contest between two teams $A=\left\{a_{1}, \cdots, a_{m}\right\}$ and $B=\left\{b_{1}, \cdots, b_{n}\right\}$, suppose first that the teams have never met before but the players of each team have played some matches among themselves. Thus there is a partial order $P$ between certain $a$ 's and certain $b$ 's, e.g., $a_{1}<a_{2}, a_{1}<a_{3}, b_{2}<b_{1}, \cdots$, but there is no direct information about the relative ranking of $a$ 's vs. $b$ 's. Denote by $\operatorname{Pr}\left(a_{1}<b_{1} \mid P\right)$ the conditional probability that $a_{1}$ loses to $b_{1}$, given the partial order $P$.

After some matches between $a$ 's and $b$ 's have taken place, in which we shall suppose that the $a$ 's have lost each match to the $b$ 's so far, we have a new partial ordering $P^{\prime}=P \cup P^{\prime \prime}$, where $P^{\prime \prime}$ contains inequalities of the form $a_{i}<b_{j}$; e.g. $P^{\prime \prime}=\left\{a_{3}<b_{4}, a_{5}<b_{2}, \cdots\right\}$. Note that there are two ways to think about $P$ : if $P$ is thought of as a partial order on $\left\{a_{1}, \cdots, a_{m}, b_{1}, \cdots, b_{n}\right\}$, then the union $P \cup P^{\prime \prime}=P^{\prime}$ is the larger partial order based on the additional information in $P^{\prime \prime}$. However, we shall think of $P$ as a subset of permutations defined by the partial order $P$ so that the intersection $P \cap P^{\prime \prime}=P^{\prime}$ is the smaller subset of permutations based on the additional information in $P^{\prime \prime}$. Denote by $\operatorname{Pr}\left(a_{1}<b_{1} \mid P^{\prime}\right)$ the conditional probability that $a_{1}$ loses to $b_{1}$ given $P^{\prime}$. It is tempting to conjecture that, in particular,

$$
\operatorname{Pr}\left(a_{1}<b_{1} \mid P^{\prime}\right) \geqq \operatorname{Pr}\left(a_{1}<b_{1} \mid P\right) .
$$

The additional knowledge with $P^{\prime}$ that $a$ 's have lost to $b$ 's prompts the belief (prejudice?) that $a$ 's are inferior to $b$ 's, and seems to make it more likely under $P^{\prime}$ than under $P$ that $a_{1}$ loses to $b_{1}$. This conjecture of R. L. Graham, A. C. Yao, and F. F. Yao [GYY] is true, as we show. However the same intuition makes it even more tempting to conclude that (1.1) holds even if $P$ contains inequalities of the form $a_{i}<b_{j}$, because the prejudice under $P$ that $a$ 's are inferior to $b$ 's is apparently further reinforced by the new inequalities in $P^{\prime}$. Nevertheless we give a simple example to show this is false. Indeed let $m=n=2$ and

$$
\begin{aligned}
& P=\left\{a_{1}<b_{2}, a_{2}<b_{1}\right\}, \\
& P^{\prime}=\left\{a_{2}<b_{2}\right\} \cap P .
\end{aligned}
$$

\footnotetext{
* Received by the editors January 14, 1980, and in final form March 11, 1980.
}

† Bell Laboratories, Murray Hill, New Jersey 07974. 
It is easy to check that $\operatorname{Pr}(P)=\frac{1}{4}, \operatorname{Pr}\left(P^{\prime}\right)=\operatorname{Pr}\left(a_{1}<b_{1}, P\right)=\frac{5}{24}, \operatorname{Pr}\left(a_{1}<\right.$ $\left.b_{1}, P^{\prime}\right)=\frac{1}{6}$ so that $(1.1)$ asserts that $\frac{4}{5} \geqq \frac{5}{6}$, which is of course false. An even simpler example was found by a referee: $m=n=2, P=\left\{a_{2}<b_{1}\right\}, P^{\prime}=\left\{a_{2}<b_{2}\right\} \cap$ $P, \operatorname{Pr}\left(a_{1}<b_{1} \mid P^{\prime}\right)=\frac{5}{8}<\frac{2}{3}=\operatorname{Pr}\left(a_{1}<b_{1} \mid P\right)$.

The example (1.2) is especially surprising because (1.1) is valid even when $P$ contains inequalities of the form $a_{i}<b_{j}$, provided that $P$ also contains inequalities which give a total ordering of each of $A$ and $B$ separately. This was proved by Graham, Yao, and Yao [GYY], and we give a new proof here.

We next give a more general formulation of the two results to be proved in $\S 2$, and discuss the FKG inequality which we will use in their proofs. Let $P_{0}$ be the subset of permutations for which $A$ and $B$ have the complete order:

$$
P_{0}=\left\{a_{1}<a_{2}<\cdots<a_{m}\right\} \cap\left\{b_{1}<b_{2}<\cdots<b_{n}\right\} .
$$

Suppose $P_{1}, P_{2}, P_{3}$ are each subsets of permutations which are intersections of subsets of the form $\left\{a_{i}<b_{j}\right\}$. Then Graham, Yao, and Yao [GYY] proved:

Theorem 1. (Graham, Yao, Yao, [GYY]).

$$
\operatorname{Pr}\left(P_{1} \mid P_{3} \cap P_{2} \cap P_{0}\right) \geqq \operatorname{Pr}\left(P_{1} \mid P_{2} \cap P_{0}\right) \text {. }
$$

Note that this is the result stated in the preceding paragraph if $P_{1}$ is specialized to a single inequality $\left\{a_{i}<b_{j}\right\}$.

Let $Q_{0}$ be a subset of permutations defined by intersections of subsets of the form $\left\{a_{i}<a_{j}\right\}$ and $\left\{b_{i}<b_{j}\right\}$ but not of the form $\left\{a_{i}<b_{j}\right\}$ or $\left\{a_{i}>b_{j}\right\}$,

$$
Q_{0}=\left\{a_{i_{1}}<a_{j_{1}}, \cdots, a_{i_{r}}<a_{j_{r}}\right\} \cap\left\{b_{k_{1}}<b_{l_{1}}, \cdots, b_{k_{s}}<b_{l_{s}}\right\},
$$

and let $P_{1}, P_{2}$ be as in Theorem 1. Then Graham, Yao, and Yao [GYY] conjectured:

Theorem 2.

$$
\operatorname{Pr}\left(P_{1} \mid P_{2} \cap Q_{0}\right) \geqq \operatorname{Pr}\left(P_{1} \mid Q_{0}\right) .
$$

The FKG (Fortuin, Kasteleyn, Ginibre) inequality was discovered [FKG] in proving "intuitively obvious" conjectures about correlations in a statistical mechanics model. Although as shown in [FKG], the FKG hypothesis $(1.7)-(1.10)$ is only sufficient for the conclusion (1.11), in the present case I found the simple counterexample (1.2) by looking for the simplest case of the general conjecture (1.1) for which the FKG technique does not easily apply. Other applications of the FKG inequality to prove known inequalities in combinatorics have been given in [SW]. D. J. Kleitman and J. B. Schearer [KS] also give an example where (1.1) fails if $P$ is allowed to contain $a_{i}<b_{j}$ inequalities, and give a different FKG proof for Theorem 1 , but do not obtain Theorem 2 .

The setting for the FKG inequality is as follows: Let $\Gamma$ be a finite lattice; i.e., $\Gamma$ is a finite set $\Gamma=\{x, y, z, \cdots\}$ with a partial order $x<y$ for which each pair $x, y \in \Gamma$ has a unique least upper bound $x \vee y$ and a unique greatest lower bound $x \wedge y$,

$$
x \vee y \in \Gamma, \quad x \wedge y \in \Gamma .
$$

Further, $\Gamma$ is assumed distributive; i.e. for all $x, y, z \in \Gamma$

$$
x \wedge(y \vee z)=(x \wedge y) \vee(x \wedge z) \text {. }
$$

or equivalently, for all $x, y, z \in \Gamma, x \vee(y \wedge z)=(x \vee y) \wedge(x \vee z)$. Suppose $\mu, f, g$ are real-valued functions on $\Gamma$ for which for all $x, y \in \Gamma$,

$$
\mu(x) \geqq 0, \quad \mu(x) \mu(y) \leqq \mu(x \wedge y) \mu(x \vee y),
$$


and $f$ and $g$ are monotonic in the same direction so that either

$$
\begin{aligned}
& f(x) \leqq f(y), \quad g(x) \leqq g(y), \text { for all } x \text { and } y, \text { or } \\
& f(x) \geqq f(y), \quad g(x) \geqq g(y), \text { for all } x<y .
\end{aligned}
$$

The FKG inequality $[\mathrm{FKG}]$ then asserts

$$
\sum_{x \in \Gamma} f(x) g(x) \mu(x) \sum_{y \in \Gamma} \mu(y) \geqq \sum_{x \in \Gamma} f(x) \mu(x) \sum_{y \in \Gamma} g(y) \mu(y) .
$$

2. Proofs of Theorems 1 and 2. Let $\Gamma$ be the set of all $\left(\begin{array}{c}m+n \\ m\end{array}\right)$ subsets of $\{1,2, \cdots$, $m+n\}$ with $m$ elements. For $x=\left\{x_{1}<x_{2}<\cdots<x_{m}\right\}, y=\left\{y_{1}<\cdots<\right.$ $\left.y_{m}\right\} \in \Gamma$, say that $x<y$ if $x_{i} \leqq y_{i}, i=1, \cdots, m$. Thus the elements of $x \wedge$ $y=\left\{(x \wedge y)_{1}<\cdots<(x \wedge y)_{m}\right\}$ and $x \vee y=\left\{(x \vee y)_{1}<\cdots<(x \vee y)_{m}\right\}$ are given for $i=1, \cdots, m$ by

$$
(x \wedge y)_{i}=\min \left(x_{i}, y_{i}\right), \quad(x \vee y)_{i}=\max \left(x_{i}, y_{i}\right) .
$$

Since $x \wedge y, x \vee y \in \Gamma,(1.7)$ holds for $\Gamma,<$.

Examining all orderings of any three real numbers $\alpha, \beta, \gamma$ shows that

$$
\min (\alpha, \max (\beta, \gamma))=\max (\min (\alpha, \beta), \min (\alpha, \gamma)) \text {. }
$$

From (2.1) and (2.2) we see that (1.8) holds so that $\Gamma,<$ is also distributive.

Let $\bar{P}_{1}, \bar{P}_{2}, \bar{P}_{3}$ each be intersections of subsets of $\Gamma$ of the form $\left\{x_{i} \leqq k\right\}$, $i=1, \cdots, m, k=1, \cdots, m+n$. Let $\mu, f, g$ be defined by

$$
\begin{aligned}
& \mu(x)= \begin{cases}1, & \text { if } x \in \Gamma \cap \bar{P}_{2}, \\
0, & \text { else, }\end{cases} \\
& f(x)=\left\{\begin{array}{ll}
1, & \text { if } x \in \Gamma \cap \bar{P}_{1}, \\
0, & \text { else, }
\end{array} \quad g(x)= \begin{cases}1, & \text { if } x \in \Gamma \cap \bar{P}_{3}, \\
0, & \text { else. }\end{cases} \right.
\end{aligned}
$$

Since $x_{i} \leqq k, y_{i} \leqq k$ implies that $\min \left(x_{i}, y_{i}\right) \leqq \max \left(x_{i}, y_{i}\right) \leqq k$, we see that $\mu(x)=\mu(y)=1$ implies that $\mu(x \wedge y)=\mu(x \vee y)=1$; thus (1.9) holds with equality. If $x \leqq y$ and $f(y)=1$ then $y \in \bar{P}_{1}$. But if $y_{i} \leqq k$ then $x_{i} \leqq k$, so that $x \in \bar{P}_{1}$ as well, and $f(x)=1$. Thus $f$ is decreasing, and similarly so is $g$. Thus (1.10) holds and the hypothesis of the FKG inequality is satisfied. By (1.11), it follows that

$$
\#\left(\Gamma \cap \bar{P}_{1} \cap \bar{P}_{2} \cap \bar{P}_{3}\right) \#\left(\Gamma \cap \bar{P}_{2}\right) \geqq \#\left(\Gamma \cap \bar{P}_{1} \cap \bar{P}_{2}\right) \#\left(\Gamma \cap \bar{P}_{2} \cap \bar{P}_{3}\right),
$$

where $\#(A)$ is the cardinality of $A$.

Consider the one-one correspondence $\phi: \Gamma \leftrightarrow P_{0}$ in (1.3); here $\phi(x)=\left(a_{1}\right.$, $\left.\cdots, a_{m}, b_{1}, \cdots, b_{n}\right)$, the permutation of $(1,2, \cdots, m+n)$ which has $a_{i}(x)=x_{i}$, $i=1, \cdots, m$, and $b_{j}(x)=j$ th element of the complement of $x$ in $\{1,2, \cdots, m+n\}$. Because the $a$ 's and $b$ 's are totally ordered by (1.3) in $P_{0}$, we have

LEMMA 2.5. If $\left(a_{1}, \cdots, a_{m}, b_{1}, \cdots, b_{n}\right) \in P_{0}$, then $a_{i}<b_{j}$ if and only if $a_{i} \leqq i+j-1$.

It follows from (2.5) that for subsets $P_{1}, P_{2}, P_{3}$ as in Theorem 1 which are each intersections of subsets $\left\{a_{i}<b_{j}\right\}, \bar{P}_{k}=\phi^{-1}\left(P_{i}\right), i=1,2,3$, are each of the form $\left\{x_{i} \leqq k\right\}$; so that (2.4) holds. Since $\phi$ is one-one we have upon dividing by $((m+n) !)^{2}$,

$$
\begin{aligned}
\operatorname{Pr}\left(P_{0} \cap P_{1} \cap P_{2} \cap P_{3}\right) & \operatorname{Pr}\left(P_{0} \cap P_{2}\right) \\
& \geqq \operatorname{Pr}\left(P_{0} \cap P_{1} \cap P_{2}\right) \operatorname{Pr}\left(P_{0} \cap P_{2} \cap P_{3}\right),
\end{aligned}
$$

which is the same as (1.4). Theorem 1 is thus proved. 
We next prove Theorem 2. For $N=1,2, \cdots$, let $\Gamma_{N}$ be the set of $N^{m+n}$ integer-valued vectors $x=\left(a_{1}, \cdots, a_{m}, b_{1}, \cdots, b_{n}\right)$ where each $a_{i}$ and $b_{j} \in\{1,2$, $\cdots, N\}$. Denote

$$
\begin{aligned}
x_{i}=a_{i}=a_{i}(x), \quad i=1, \cdots, & \\
& x_{m+j}=b_{j}=b_{j}(x), \quad j=1, \cdots, n .
\end{aligned}
$$

For $x, y \in \Gamma_{N}$ say that $x<y$ if $x_{i}=a_{i}(x) \leqq a_{i}(y)=y_{i}$ but $x_{m+j}=b_{j}(x) \geqq b_{j}(y)=$ $y_{m+j}, j=1, \cdots, n$. The components of $x \wedge y$ and $x \vee y$ are

$$
\begin{array}{ll}
(x \wedge y)_{i}=\min \left(x_{i}, y_{i}\right), & (x \wedge y)_{m+j}=\max \left(x_{m+j}, y_{m+j}\right), \\
(x \vee y)_{i}=\max \left(x_{i}, y_{i}\right), & (x \vee y)_{m+j}=\min \left(x_{m+j}, y_{m+j}\right) .
\end{array}
$$

Since $x \wedge y, x \vee y \in \Gamma_{N},(1.7)$ holds for $\Gamma_{N}$.

Because of (2.2), we again have (1.8) so $\Gamma_{N},<$ is also a finite distributive lattice.

Let $Q_{0}^{*}$ be a subset of $\Gamma_{N}$ defined by intersections of subsets of the form $\left\{x: a_{i}(x)<a_{j}(x)\right\}$ and $\left\{x: b_{i}(x)<b_{j}(x)\right\}$, so that

$$
\begin{aligned}
Q_{0}^{*}=\{x: & a_{i_{1}}(x)<a_{j_{1}}(x), \cdots, a_{i_{r}}(x)<a_{j_{r}}(x) ; \\
& \left.b_{k_{1}}(x)<b_{l_{1}}(x), \cdots, b_{k_{s}}(x)<b_{l_{s}}(x)\right\},
\end{aligned}
$$

and let $P_{1}^{*}$ and $P_{2}^{*}$ be subsets of $\Gamma_{N}$ defined by intersections of the form $\left\{x: a_{i}(x)<b_{j}(x)\right\}$. Let $\mu, f, g$ be defined for $x \in \Gamma_{N}$ by

$$
\begin{aligned}
& \mu(x)= \begin{cases}1, & \text { if } x \in Q_{0}^{*}, \\
0, & \text { else, }\end{cases} \\
& f(x)=\left\{\begin{array}{ll}
1, & \text { if } x \in P_{1}^{*}, \\
0, & \text { else, }
\end{array} ; \quad g(x)= \begin{cases}1, & \text { if } x \in P_{2}^{*}, \\
0, & \text { else. }\end{cases} \right.
\end{aligned}
$$

If $x, y \in \Gamma_{N}$ and $\mu(x) \mu(y)=1$, then $x, y \in Q_{0}^{*}$ so that for $z=x$ or $y, a_{i_{t}}(z)<a_{j_{t}}(z), t=1, \cdots, r$, and $b_{k_{t}}(z)<b_{t_{t}}(z), t=1, \cdots, s$. But then

$$
\begin{aligned}
& \min \left(a_{i_{t}}(x), a_{i_{t}}(y)\right)<\min \left(a_{j_{t}}(x), a_{j_{t}}(y)\right), \quad t=1, \cdots, r, \\
& \max \left(b_{k_{t}}(x), b_{k_{t}}(y)\right)<\max \left(b_{l_{t}}(x), b_{l_{t}}(y)\right), \quad t=1, \cdots, s,
\end{aligned}
$$

so that by (2.7) and (2.8), $x \wedge y \in Q_{0}^{*}$. Similarly, $x \vee y \in Q_{0}^{*}$, so that $\mu(x \wedge y) \mu(x \vee y)=1$. Thus (1.9) holds. Note that (1.9) would fail if $Q_{0}^{*}$ were allowed to contain inequalities $\left\{a_{i}<b_{j}\right\}$.

If $x<y$ and $f(y)=1$, then $y \in P_{1}^{*}$; so that $a_{i}(x) \leqq a_{i}(y) \leqq b_{j}(y) \leqq b_{j}(x)$ if $\left\{a_{i}<b_{j}\right\}$ is any one of the inequalities involved in $P_{1}^{*}$. Thus $x \in P_{1}^{*}$, and so $f(x)=1$. Thus $f(x)$ is decreasing and so is $g$. Thus (1.10) holds and the hypothesis of the FKG inequality is satisfied. By (1.11) it follows that

$$
\#\left(Q_{0}^{*} \cap P_{1}^{*} \cap P_{2}^{*}\right) \#\left(Q_{0}^{*}\right) \geqq \#\left(Q_{0}^{*} \cap P_{1}^{*}\right) \#\left(Q_{0}^{*} \cap P_{2}^{*}\right) .
$$

Now consider the subset $\Gamma_{N}^{*}$ of $\Gamma_{N}$, for which all $a_{i}$ 's and $b_{j}$ 's are distinct. Since $\#\left(\Gamma_{N}^{*}\right)=N(N-1) \cdots(N-n-m+1)$, we see that

$$
\lim _{N \rightarrow \infty} \frac{\#\left(\Gamma_{N}^{*}\right)}{\#\left(\Gamma_{N}\right)}=1 \text {. }
$$

In $\Gamma_{N}^{*}$ however, $a_{1}(x), \cdots, a_{m}(x), b_{1}(x), \cdots, b_{n}(x)$ are all distinct, and for each $x \in \Gamma_{N}^{*}$ a unique ordering of $a_{1}, \cdots, a_{m}, b_{1}, \cdots, b_{n}$ is obtained by letting the ordering of $a_{i}(x), b_{j}(x)$ determine the ordering of $a_{i}, b_{j}$. For $N \geqq n+m$, the fraction of 
$\Gamma_{N}^{*}$ corresponding to each ordering of $a_{1}, \cdots, a_{m}, b_{1}, \cdots, b_{n}$ is $1 /(m+n)$ !; so that for $N \geqq m+n$,

$$
\frac{\#\left(A^{*} \cap \Gamma_{N}^{*}\right)}{\#\left(\Gamma_{N}^{*}\right)}=\operatorname{Pr}(A)
$$

Here $A=Q_{0}, Q_{0} \cap P_{i}, Q_{0} \cap P_{1} \cap P_{2}$ given in Theorem 2 and in (1.5), and the corresponding $A^{*}=Q_{0}^{*}, Q_{0}^{*} \cap P_{i}^{*}, Q_{0}^{*} \cap P_{1}^{*} \cap P_{2}^{*}$. Since for any $A$ and the corresponding $A^{*}$ we have

$$
\#\left(A^{*} \cap\left(\Gamma_{N}-\Gamma_{N}^{*}\right)\right) \leqq \#\left(\Gamma_{N}-\Gamma_{N}^{*}\right)=o\left(\#\left(\Gamma_{N}^{*}\right)\right),
$$

as $N \rightarrow \infty$, it follows that also

$$
\lim _{N \rightarrow \infty} \frac{\#\left(A^{*}\right)}{\#\left(\Gamma_{N}^{*}\right)}=\operatorname{Pr}(A)
$$

for $A=Q_{0}, Q_{0} \cap P_{i}, Q_{0} \cap P_{1} \cap P_{2}$, and $A^{*}=Q_{0}^{*}, Q_{0}^{*} \cap P_{i}^{*}, Q_{0}^{*} \cap P_{1}^{*} \cap P_{2}^{*}$, respectively. Thus from (2.12) and (2.16) we obtain, letting $N \rightarrow \infty$,

$$
\operatorname{Pr}\left(Q_{0} \cap P_{1} \cap P_{2}\right) \operatorname{Pr}\left(Q_{0}\right) \geqq \operatorname{Pr}\left(Q_{0} \cap P_{1}\right) \operatorname{Pr}\left(Q_{0} \cap P_{2}\right),
$$

which is the same as (1.6). Theorem 2 is thus proved.

\section{REFERENCES}

[1] [FKG]. C. M. Fortuin, P. W. Kasteleyn, And J. Ginibre, Correlation inequalities on some partially ordered sets, Comm. Math. Phys., 22 (1970), pp. 89-103.

[2] [GYY]. R. L. Graham, A. C. Yao, And F. F. Yao, Some monotonicity properties of partial orders, this Journal, this issue, pp. 251-258.

[3] [KS]. D. J. Kleitman and J. B. Schearer, Some monotonicity properties of partial orders, Studies in Appl. Math., to appear.

[4] [SW]. P. D. SEymour AND D. J. A. Welsh, Combinatorial applications of an inequality from statistical mechanics, Math. Proc. Cambr. Philos. Soc., 77 (1975), pp. 485-495. 